\title{
Design of crushable particles in DEM based on single grain compression
}

\author{
Bruna Tedesco ${ }^{1,{ }^{*}}$, Manoel Porfirio Cordão $\mathrm{Neto}^{1}$, Alessandro Tarantino $^{2}$, and Márcio Farias ${ }^{1}$ \\ ${ }^{1}$ University of Brasilia (UnB), Civil Engineering Department, 70910-900 Brasília, Brazil \\ ${ }^{2}$ University of Strathclyde, Civil and Environmental Engineering Department, Glasgow, Scotland, UK
}

\begin{abstract}
Grain crushing controls the response in shear of granular materials and has an impact on the collapse mechanism of geo-structures. To simulate the effects of grain crushing on the macroscopic response, the Discrete Element Method (DEM) appears to be a viable option considering its proven capability of capturing key aspects of the response of granular materials. One of the approaches to simulate particle crushing via DEM consists in creating a particle by assembling smaller sub-particles 'bonded' together by adhesion forces. When the force at the contact between two sub-particles exceeds the adhesion, the bonding is broken irreversibly. A critical aspect in this approach is the characterization of i) the 'random' distribution of the adhesion between the sub-particles, and ii) the initial rotation angle of the particles being assembled initially. The tensile strength of a single grain, as measured experimentally by diametral compression between flat platens, typically follows the Weibull distribution. This is proposed to be the criterion to validate any probabilistic distribution selected for the adhesion and the initial rotation angle. The effect of a normal distribution of adhesion and initial rotation on compressive strength was investigated by compressing either a regular 'cylindrical' particle or an irregular particle. It is shown that a normal probabilistic distribution of both the adhesion and the rotation angle returns a Weibull distribution of the compressive strength. It is also shown that the rotation angle plays a very critical role and this should be taken into account when initially assembling the 'crushable' particles. The rotation angle relocates the contact points, which in turn control the stress at which the particle breaks.
\end{abstract}

\section{Introduction}

Grain crushing controls the response in shear of granular materials and therefore has an impact on the collapse mechanism of geo-structures [1]. It is a critical of soil behaviour and is a result of a number of relatively complex micro-processes.

It is commonly accepted that the crushing of a grain occurs due to a tensile failure and its tensile strength, responsible for the particle crushing, can be indirectly measured by diametral compression [2]. A variety of compression testing methods can be used to measure the breakage characteristics of single particles. Each test method allows investigations over a restricted range of deformation rates [3]. Slow compression tests conducted by placing an individual particle between two flat and applying a uniaxial compression to the particle until fracture are often used. The main advantage of this technique is the possibility of recording the deformations in order to determine the load-deformation profile.

The Weibull statistical distribution is widely accepted as a probabilistic representation of the tensile strength of brittle ceramics. The Weibull distribution assumes that the survival of a block of a material under tension requires that all its constituent part remains intact. From this, Weibull determines the survival probability. This probability takes into account the particle diameter and nature of the material forming the block [2].

It is known that there are a number of specific micro characteristics that influence the way and the time at which the particle breaks. These include the presence of critical flaws and cracks in zones of high stress in the particle. Thus, the bigger the particles, the higher is the likelihood to find flaws and cracks in the zones of high stress and, hence, the higher is the susceptibility to breakage [5]. Accordingly, the increase in particle size results in a shift of the resistance to lower values.

Besides that, grains having similar shape and size can also show different tensile strength values. The geological material from which particles originated is characterised by defects and cracks. When particles are formed from it, cracks and defects will be distributed differently particle by particle resulting in different values of tensile strength.

The shape of the particle is another characteristic that influences breakage. Particle shape directly influences the state of stresses behaviour inside the particle. The particle strength and its stiffness decrease significantly as particle shape becomes more irregular [6]. In principle, the orientation of irregular particle with respect to the loading point also affects its susceptibility to failure. Particles having high sphericity should show low variability in

\footnotetext{
Corresponding author: bmotams@gmail.com
} 
contrast with the particle having low sphericity. Sphericity can also change with the size of the particle.

Several aspects of the response of granular materials can be adequately captured by the Discrete Element Method (DEM), which makes this method one of the best numerical approaches for simulating the response of granular materials. The DEM also allows a better understanding of the response of granular materials at the particle scale and its interplay with macroscopic response. The DEM therefore becomes an almost obvious option to represent and analyse grain crushing in granular materials.

There are two main approaches implemented to analyse the response of 'crushable' particles using DEM. One possible approach, referred to as the agglomerate method, consists in creating a particle by assembling a large number of smaller sub-particles 'bonded' together by adhesion forces. When the force at the contact between two sub-particles exceeds the adhesion, the bonding is irreversible broken. As breakages occurs, the micro particles separate from its original particle, representing fragments. Another approach consists of replacing the original particle by smaller particles when a limit tension value is reached [7].

For the agglomerate method, one needs to design the agglomerate, i.e. its shape and the distribution of bonding at the contacts (by either assuming a distribution of adhesion or introducing defects). The approach pursued in this paper is to design the agglomerate in order to match the Weibull distribution of tensile strength based experimental crushing test on a single grain. There are aspects affecting the survival probability of the agglomerate that have not been investigated in depth. Understanding what generates a Weibull distribution of compressive strength is clearly critical in the design of the agglomerates. This would allow identifying the critical geometrical and material parameters that need to be calibrated against the experimental observation (e.g. diametral compression test).

This paper presents an investigation of some aspects of a particle breakage using a particle formed by micro spheres bonded together by adhesion forces. It explores the role of the 'random' distribution of the adhesion between the sub-particles, and of the initial rotation angle of the particles being assembled initially. Random distributions of adhesion and rotation angle benchmarked against the Weibull distribution of tensile strength expected to be returned by DEM simulations of diametral compression test on individual crushable grains.

\section{Methodology}

In order to analyse aspects that influences the crushing processes of a particle, different virtual tests were carried out. To analyse the influence of the particle internal variation of resistance in the crushing process, a first virtual test on a 'cylindrical' particle compressed between two flat platens was carried out. The particle is formed by 13,000 smaller spherical sub-particles 'bonded' together as shown in Fig. 1. Bonding between the sub-particles is represented by adhesion forces that follow a normal distribution of probability (mean equal to $100 \mathrm{kPa}$ and standard deviation equal to $50 \mathrm{kPa}$ ). As the particle is compressed, the forces between spheres increase. The limit for this growth is the adhesion between them. If the force is greater than the adhesion, the two sub-particles loose the bonding between them. The 'cylindrical' particle eventually splits when the unbonded contacts form a continuous surface throughout the particle.

The compression test was repeated 200 times maintaining the same particle with the same sub-particles positions and the same displacement rate of the top platen. The only variation imposed was the distribution of adhesion between the particles (though sampled from the same normal distribution).

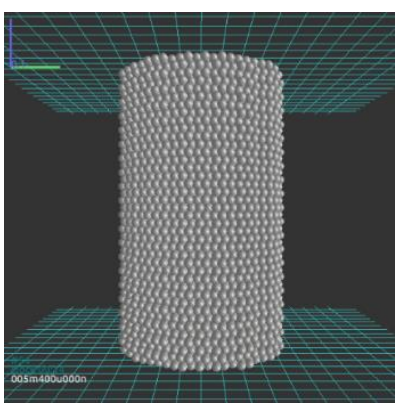

Fig. 1. Cylindrical Particle.

The manner in which a particle is oriented in a compression test also determines its breakage behaviour. To investigate this aspect, a second series of virtual tests was carried out. An irregularly shaped particle was created by assembling spherical sub-particles 'bonded' together by adhesion forces. The shape created for the virtual compression mimicked the real shape of a sand grain as shown in Fig. 2.

In this series of tests, the particle shape, the adhesion between particles, and displacement rate of the top platen were maintained the same. The rotational angle of the particle with respect to the $\mathrm{x}$-axis was varied according to a normal distribution (mean value equal to $90^{\circ}$ and standard deviation equal to $30^{\circ}$ ).
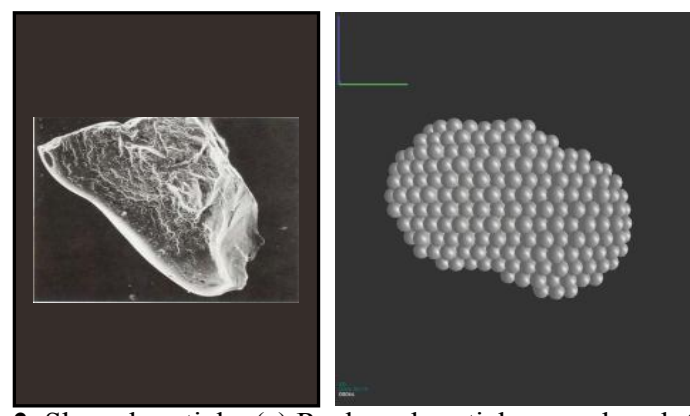

Fig. 2. Shaped particle. (a) Real sand particle reproduced. (b) Virtual sand particle

The compression test was repeated 200 times to obtain a statistically representative dataset. The rotation angles imposed in the 200 tests and extracted from a normal distribution. It is important to notice that, differently from the previous test, the particle now is confined by 6 planes during the compression as shown in Fig. 3. This confinement is important to maintain the same inclination up to failure. If the particle was compressed by two 
platens only, the particle would first rotate rigidly until a new contact point ensures zero moment on the particle.

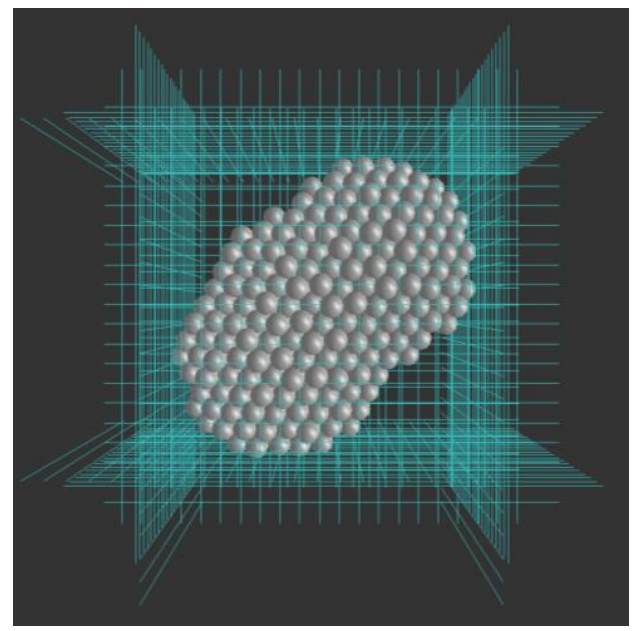

Fig. 3. Confinement planes

In the third series of tests, the same shaped particle (Fig. 2) was compressed. However, the particle orientation was maintained fixed and the distribution of adhesion between sub-particles was varied according to the same probabilistic distribution as in the first series. One more time, all the other parameters of the simulation were maintained constant and, this time, the simulation was repeated 100 times.

Finally, the effect of random particle orientation and adhesion between sub-particles were analysed together in the fourth series of tests.

The material parameters used in all simulations were the same. A value of $500 \mathrm{kPa}$ was used for the particle Young's modulus. The Poisson's ratio of the particles was set equal 0.3 and the density of the grain was set to 2600 $\mathrm{kg} / \mathrm{m} 3$. The size of the spherical sub-particles was set to be $0.015 \mathrm{~m}$.

\section{Results}

The results from the four series of compressions tests are shown in Fig. 4 in terms of vertical force vs vertical displacement. The peak in the force-displacement curve represents the 'strength of the particle'.

The variability of particle strength is associated with the randomness of the adhesion forces between subparticles and/or the orientation of the particle. For example, different distributions of adhesion forced in the cylindrical particle give rises to different strengths as shown in Fig. 4a. Accordingly, these also generated different modes of crushing in the compression tests as shown Fig. 5a, i.e. variations in the adhesion distributions generates distinct weakness surfaces within the particle.

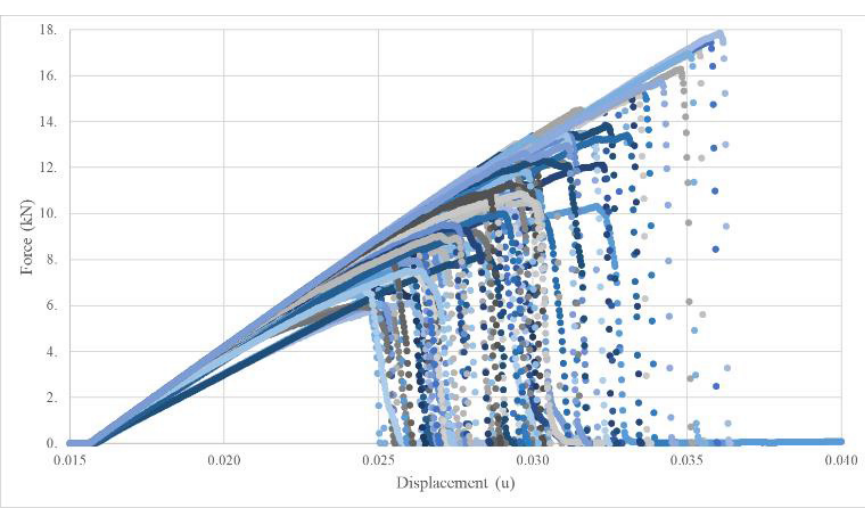

(a)

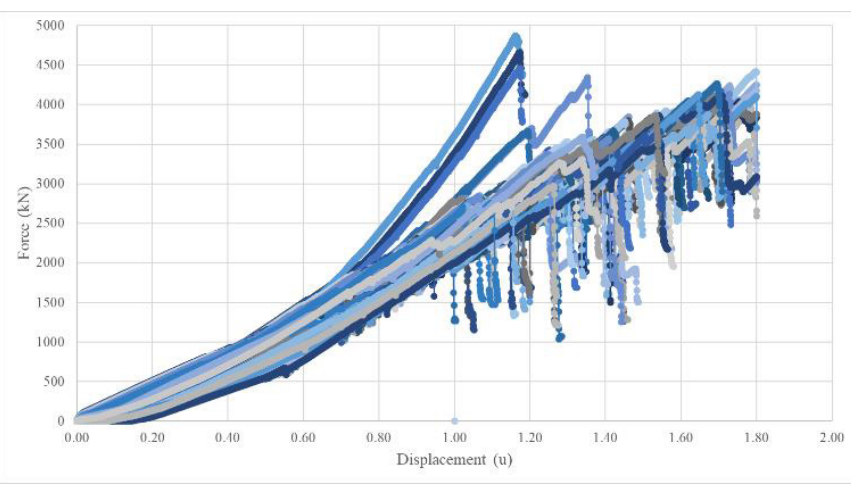

(b)

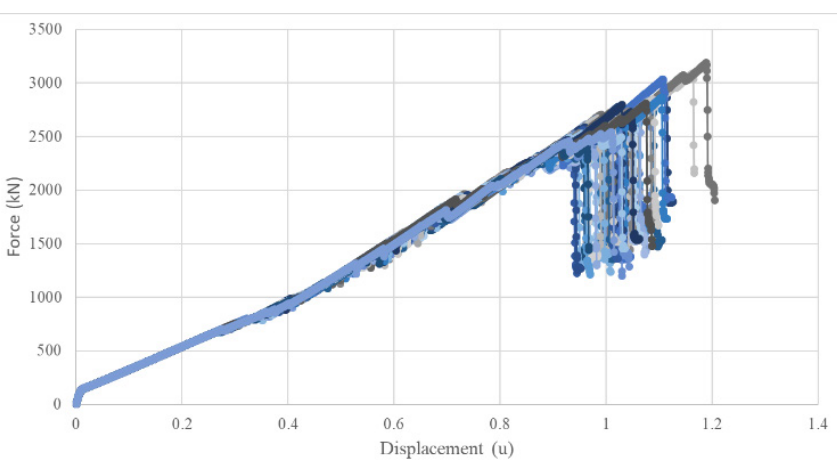

(c)

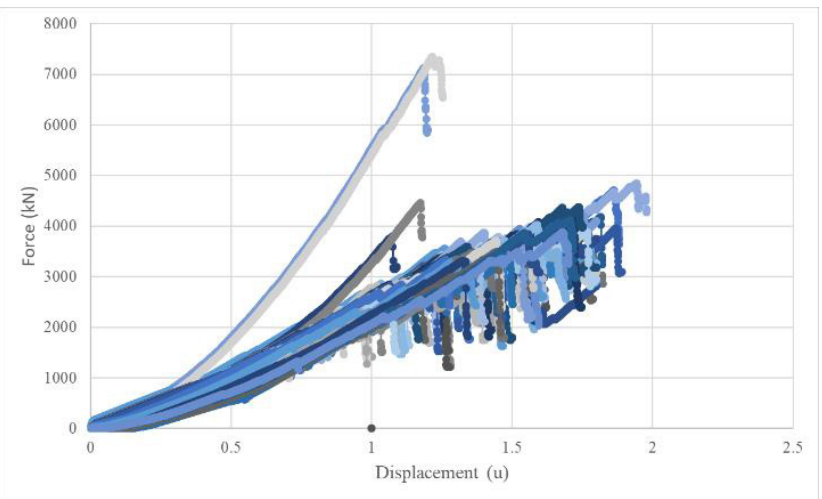

(d)

Fig. 4. Compression Tests: (a) Adhesion distribution test on a cylindrical particle; (b) Rotation distribution test on a shaped particle; (c) Adhesion distribution test on a shaped particle; (d) Adhesion and Rotation distribution test on a shaped particle. 
The effect of the rotation angle on the particle strength is shown in Fig. $4 \mathrm{~b}$ and an example of the different modes of crushing observed is presented in Fig. 5b. The difference in the crushing behaviour can now be attributed to different configuration of internal forces generated by the relocation of the 6 contact points as the rotation angle is varied.

Finally, Fig. 4c shows the effect of random adhesion on the particle maintaining the same orientation and Fig. $4 \mathrm{~d}$ shows the combined effect of adhesion and rotation angle randomness.

It is worth noticing that the order of magnitude of the compression strength 'measured' in the cylindrical and irregularly shaped particle is significantly different. This is associated with the different coordination number, equal to 2 and 6 for the cylindrical and irregularly shaped particle respectively.

It is also worth noticing that all the curves in Fig. 4 present, before the final 'catastrophic' crushing, there are small crushing events evidenced by the drop in compressive force. This fact can be explained by the work inputted by the top platen only partially released as kinetic energy of the sub-particles, with the remaining work input still stored as elastic energy [3].
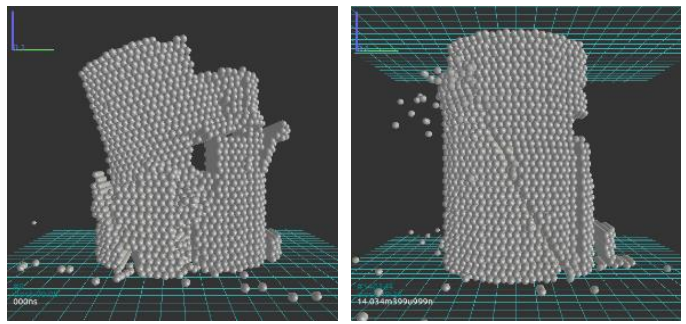

(a)
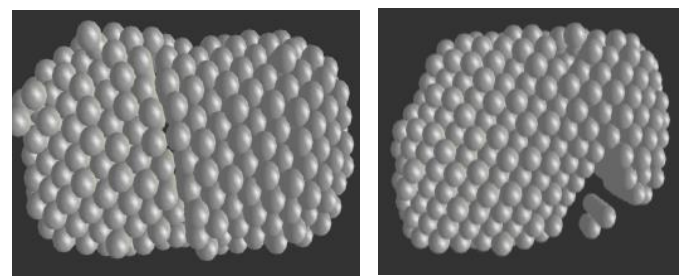

(b)

Fig. 5 Different modes of particle crushing: (a) Adhesion distribution test on a cylindrical particle; (b) Rotation distribution test on a shaped particle.

The frequency distribution of the particle strength measured in four series of virtual tests is shown in Fig. 6. The distribution is approximately normal and this is not surprising as they originate from a normal distribution of adhesion forces between sub-particles and/or particle rotation angle.

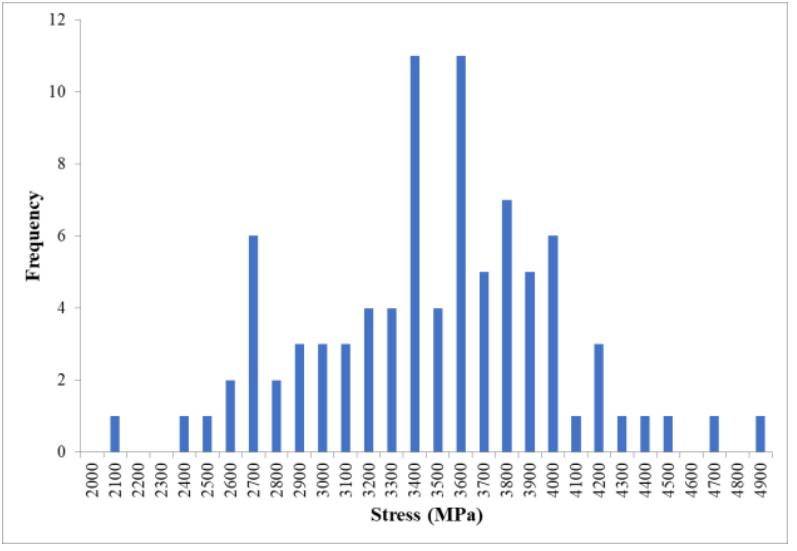

(a)

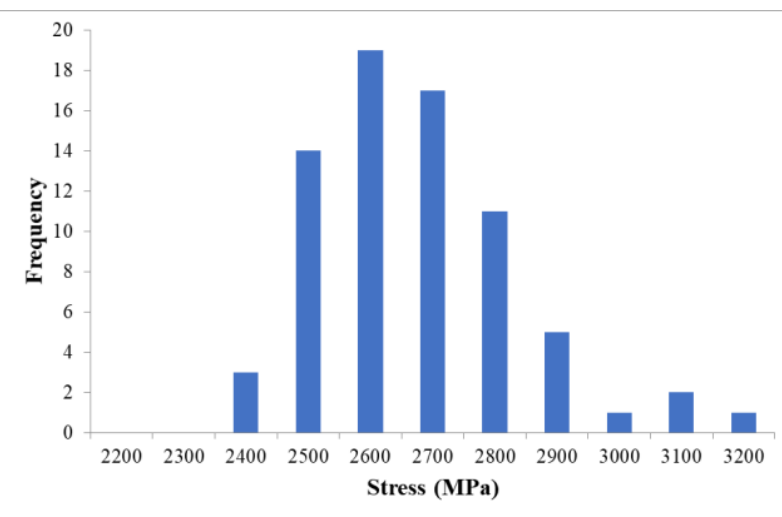

(b)

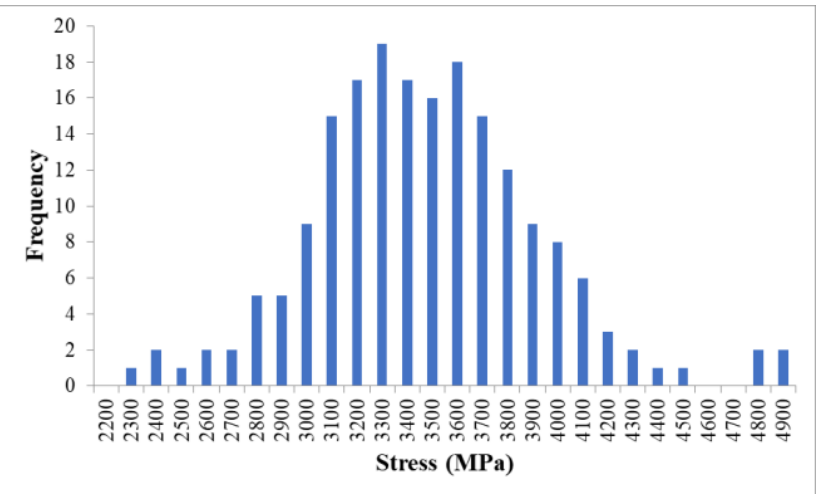

(c)

Fig. 6 Maximum stress distribution: (a) Rotation distribution test on a shaped particle; (b) Adhesion distribution test on a shaped particle; (c) Adhesion and rotation distribution test on a shaped particle.

\section{Data interpretation}

The tensile strength of individual grains measured experimentally in diametral compression tests is generally found to be represented by the Weibull distribution. The Weibull distribution provides the survival probability of a particle.

The distribution of the survival probability of the particles compressed in the virtual tests discussed above can then be inferred from the probabilistic distribution of compressive strength shown in Fig. 6. 
The criterion to validate the statistical distribution adopted for any of the parameters of the 'crushable' particle (including the adhesion between sub-particle and the initial particle rotation) is based on their capability to return a Weibull distribution of the tensile strength in DEM simulations of 'crushable' particle compression.

Fig. 7 shows indeed that the Weibull distribution fits reasonably well the distribution of survival probability in each of the four series of virtual tests carried out (Table 1 summarizes all the parameters used to calibrate the Weibull distributions). It is therefore inferred that a normal distribution of adhesion forces and/or initial rotation angles are suitable to represent the 'crushable' behaviour of a single 'aggregate' particle.

Fig. 8 shows the comparison between the survival probability distributions derived from the virtual tests on the irregularly shaped particle (the distribution associated with the 'cylindrical' particle is not shown in this figure because is characterized by values of particle strength of two orders of magnitude smaller).

Inspections of the $\mathrm{m}$ parameter of the Weibull distribution reveals that the values derived from the virtual tests fit the expected values for soils, which should range between 5 and 10 according to McDowell and Bolton (1998). The only exception is represented by the case of the irregularly shaped particle with random adhesion forces between sub-particles where the $\mathrm{m}$ parameter appears to be much higher. This can also be observed in Fig. 8 as the distribution for the irregularly shaped particle with random adhesion forces is much steeper.

This can either suggest that the standard deviation for the adhesion force distribution was selected too small or that the adhesion force distribution plays a minor role in generating the Weibull distribution of survival probability.

Table 1. Parameters for the Weibull distributions.

\begin{tabular}{ccc}
\hline & $\sigma_{0}(\mathrm{MPa})$ & $\mathrm{m}$ \\
\hline $\begin{array}{c}\text { Adhesion distribution test in a } \\
\text { cylindrical particle }\end{array}$ & 11.2 & 5 \\
\hline $\begin{array}{c}\text { Rotation distribution test in a } \\
\text { shaped particle }\end{array}$ & 3600 & 9 \\
\hline $\begin{array}{c}\text { Adhesion distribution test in a } \\
\text { shaped particle }\end{array}$ & 2650 & 27 \\
\hline $\begin{array}{c}\text { Adhesion and rotation } \\
\text { distribution test in a shaped } \\
\text { particle }\end{array}$ & 3600 & 8 \\
\hline
\end{tabular}

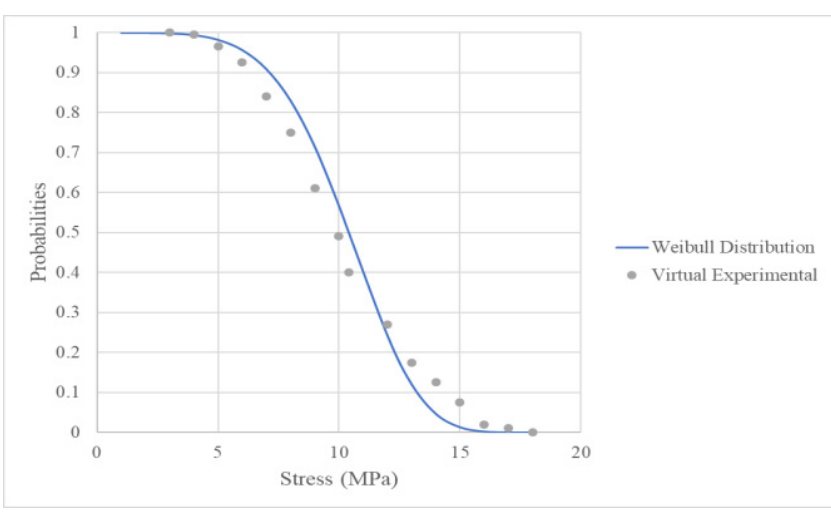

(a)

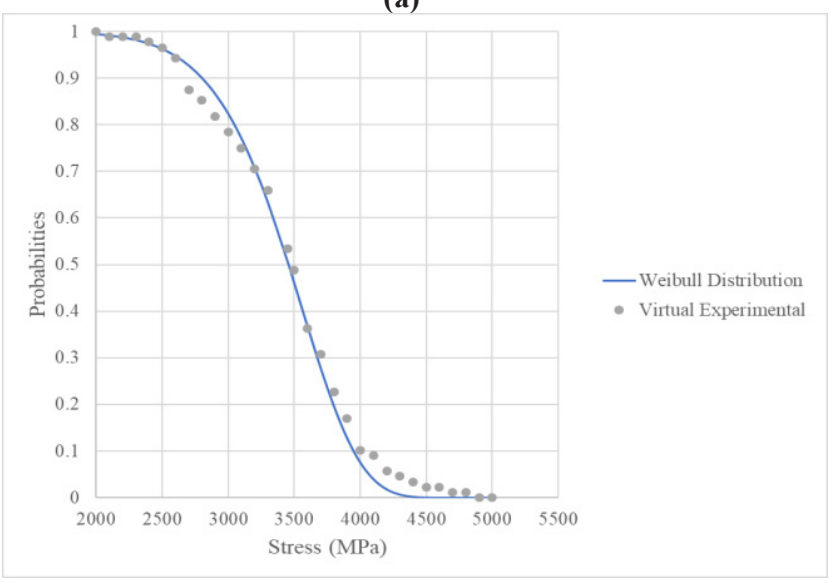

(b)

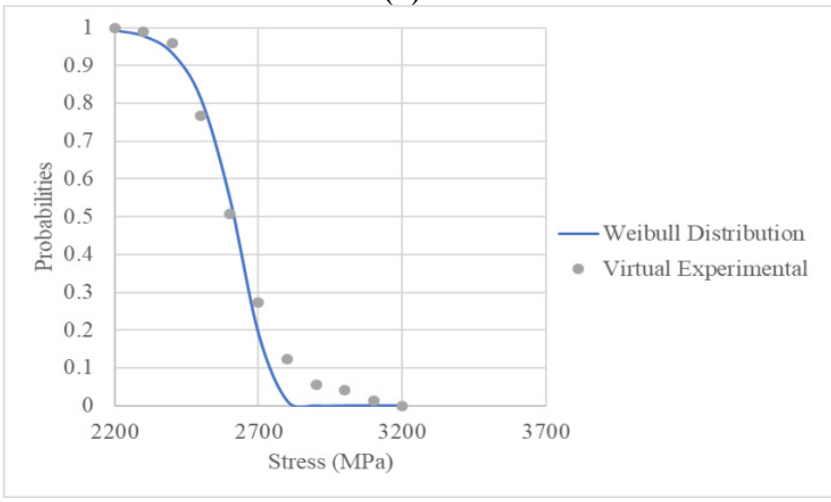

(c)

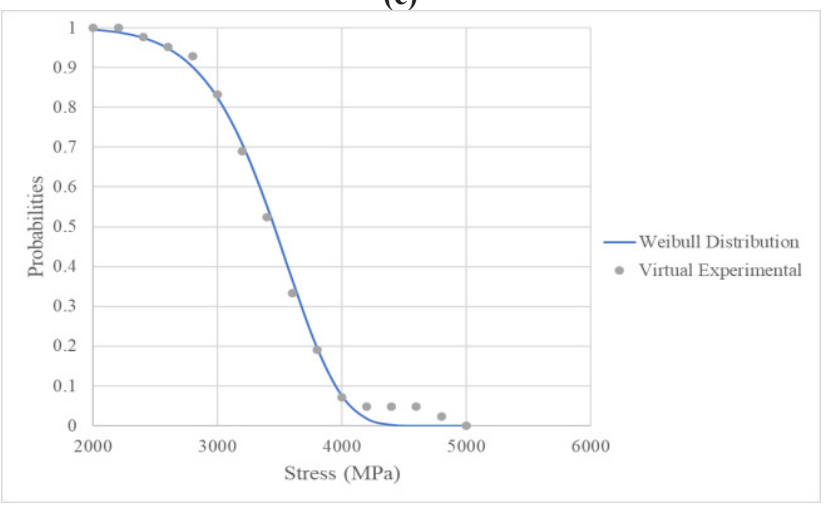

(d)

Fig. 7 Weibull and experimental distributions: (a) Adhesion distribution tests in a cylindrical particle; (b) Rotation distribution tests in a shaped particle; (c) Adhesion distribution test in a shaped particle; (d) Adhesion and rotation distribution test in a shaped particle. 
Nonetheless, Fig. 8 shows that the rotation angle of the particle plays a very significant role as it returns realistic values of the $m$ parameter as measured experimentally. The lesson learned from this sets of numerical experiments is therefore that the initial rotation angle to be assigned to individual 'crushable' particles when assembling the sample should also be characterised by a given probabilistic distribution.

This is because the rotation angle affects the dislocation of the contact points which in turn affects the distribution of internal mobilised forces within the particle, i.e. between the sub-particles. This finding suggests that the shape of the particle also plays a critical role in the modelling of the assemblage of crushable particles, although this aspect has not been investigated explicitly in this paper.

Finally, the tests performed on the irregularly shaped particle with random adhesion forces and inclination, have shown that the adhesion plays a minor role on the survival probability. Nonetheless, the adhesion between sub-particles participates in the definition of the manner in which the weakness surface forms within the particle until it eventually splits.

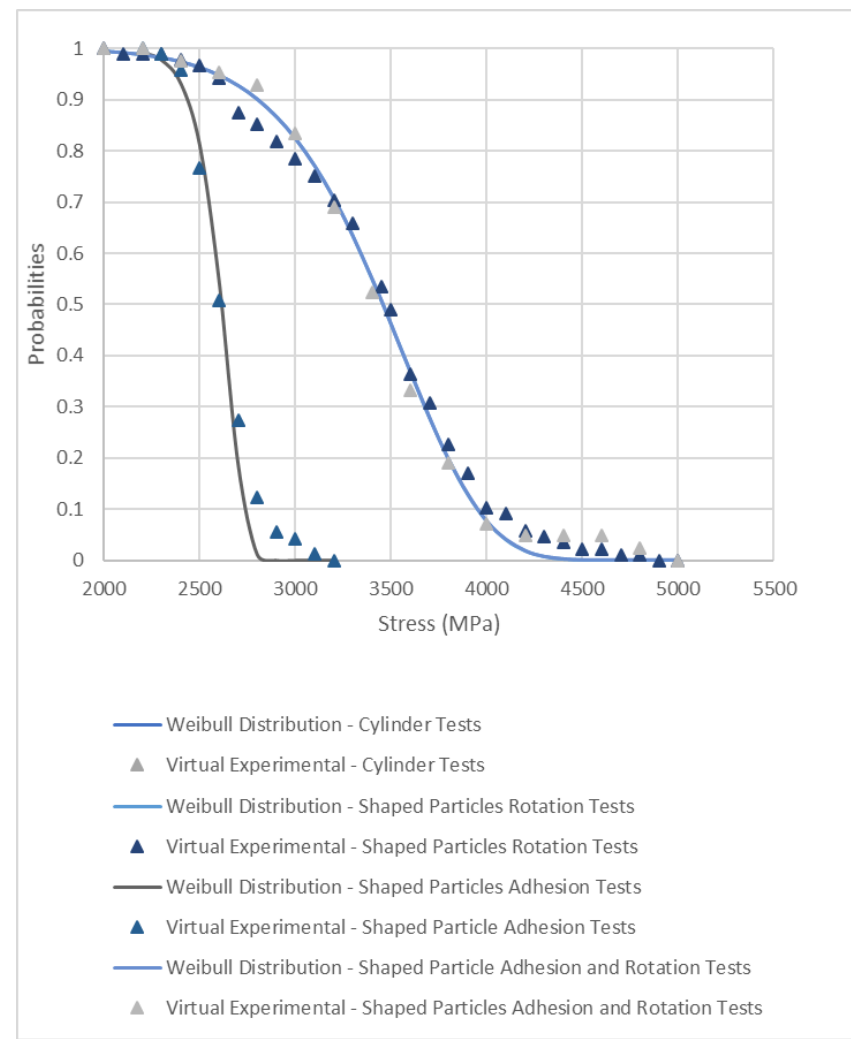

Fig 8 Comparisons of Weibull and Experimental Distributions

\section{Conclusions}

The paper has shown that a normal distribution of adhesion and initial rotation angle in a 'crushable' particle appears to return successfully a Weibull distribution of particle tensile strength. This type of distribution can therefore be used in the design of 'crushable' particles within the 'agglomerate' approach. It is also shown that the rotation angle plays a very critical role and this should be taken into account when initially assembling the 'crushable' particles. The rotation angle relocates the contact points, which in turn control the stress at which the particle breaks. It can finally be inferred that the shape of the particle should also play a critical role as the shape controls the position of the contact points in a very similar way.

The authors wish to acknowledge the support of the European Commission via the Marie Curie IRSES project GREAT 'Geotechnical and geological Responses to climate change: Exchanging Approaches and Technologies on a world-wide scale' (FP7-PEOPLE-2013-IRSES-612665).

\section{References}

1. A. Tarantino, A. F. L. Hyde, An experimental investigation of work dissipation in crushable materials, Géotechnique 55, 8, 575 - 584 (2005).

2. G. R. McDowell, M. D. Bolton. On the micromechanics of crushable aggregates. Géotechnique 48, 5, 667-679 (1998).

3. L. M. Tavares. Breakage of Single Particles: QuasiStatic. Department of Metallurgical and Materials Engineering, Universidade Federal do Rio de Janeiro, Brazil (2007).

4. T. Rasp, T. Kraft, H. Ridel. Discrete element study on the influence of initial coordination numbers on sintering behaviour, Elsevier (2013).

5. E. E. Alonso, M. Tapias, J. Gili. Scale effects in rockfill behavior. Géotechnique Letters 2 (2012).

6. L. M. Tavares, R. P. King. Int. J. Miner. Process. 54 (1998) 1.

7. B. M. M. S. Tedesco, Modelagem Numérica de Ensaios de Enrocamento, Dissertação de Mestrado, Universidade de Brasília (2016). 